\title{
Medicina y filosofía: abordaje filosófico de algunos problemas de la medicina actual
}

\author{
Adolfo Peña ${ }^{1}$ \\ ${ }^{1}$ Clínica Apóstol Juan Pablo, Lima, Perú.
}

\begin{abstract}
Resumen
El pensamiento médico y el ejercicio de la medicina plantean un cúmulo de problemas filosóficos. Éstos estriban desde la caracterización de la medicina hasta los problemas epistemológicos, lógicos y éticos. Este ensayo abordará filosóficamente seis problemas de la medicina actual: la investigación biomédica -su evolución actual y las implicancias de las políticas de desarrollo científico-; la medicina basada en evidencias -su caracterización como nuevo paradigma y el análisis de su "verdadero" rol en la práctica médica-; la caracterización de la medicina como saber y praxis - ¿qué entendemos por medicina, arte, ciencia o tecnología?; el problema de la falta de un concepto general de enfermedad; la epistemología personal y sus implicancias en la labor del médico; por último, el examen filosófico sobre la validez de la llamada medicina alternativa. Este ensayo pretende mostrar que el abordaje filosófico de muchos problemas médicos no representa un mero juego intelectual, sino una herramienta eficaz para la investigación y la enseñanza de la medicina, principalmente, para la observación crítica de la problemática en juego. Se pretende mostrar, además, que la filosofía de la medicina no puede resumirse sólo a la parcela de lo ético, lo axiológico o lo histórico.
\end{abstract}

Palabras clave: Filosofía médica; investigación; enfermedad; práctica profesional; medicina alternativa.

Medicine and philosophy: philosophical addressing of some problems of today's medicine Summary

The medical thought and practice pose a number of philosophical problems; these include the characterization of medicine itself, and the epistemological, ethical and logic problems. This essay will philosophically address six current medical problems: The biomedical investigation -evolution today and the implication of the scientific development policies-; the evidence based medicine -its characterization as new paradigm, and the analysis of its "real" role in medical practice-; the characterization of medicine as knowledge and practice -What do we understand by medicine, art, science or technology?-; the problem of lack of a general disease concept -the personal epistemology and its implications in the physicians' work-; finally, the philosophical examination on the validity of so named alternative medicine. This essay intends to show that the philosophical addressing of many medical problems does not represent a mere intellectual game, but an effective tool for medical research and teaching, mainly, for the critical observation of problems. We intend to show, furthermore, that the philosophy of medicine cannot be summarized only to the ethical and historical plot.

Key words: Philosophy, medical; research; disease; professional practice; alternative medicine.

Tras cuatro siglos de desarrollo científico, y casi dos siglos de medicina basada en la investigación científica $\left(^{1}\right)$, pareciese que la medicina ha avanzado ya lo suficiente. Actualmente, poseemos profusa información acerca de los diferentes agentes etiológicos y acerca de las más novedosas herramientas diagnósticas y terapéuticas. Cada año, la investigación biomédica publica miles de artículos; tantos, que

\footnotetext{
Correspondencia:

Dr. Adolfo Peña Salazar

Choquehuanca 208

Lima 32, Perú.

E-mail:adolfoinquiry@yahoo.com
} 
se les calcula en once millones los publicados hasta la actualidad $\left({ }^{2,3}\right)$. Estamos, pues, copados de información y de datos; pareciese, entonces, que casi todo está dicho y hecho, hasta el punto de no poder encontrar materia libre de investigación. Sin embargo, si avistáramos con mayor espíritu crítico, podríamos revelar que tal avance puede ser insuficiente e incluso aventurado. Por ejemplo, un estudio ha mostrado que la literatura médica es caótica en lo que respecta a su organización $\left(^{4}\right)$; muchas revistas compiten por el mismo material, siendo su área de especialización muy estrecha, sin poder cubrir adecuadamente las necesidades de todos los involucrados en el cuidado del paciente.

Desde hace décadas, el paradigma de la investigación biomédica ha enfatizado el desarrollo de un cuidadoso balance entre la investigación básica y la investigación clínica $\left(^{5}\right)$. Sin embargo, estudios recientes sobre el desarrollo científico del país más rico del mundo muestran que actualmente ese balance ya no se mantiene. En esa coyuntura, los médicos-científicos están desapareciendo como investigadores principales ( ${ }^{6}$ ); muchos de los puestos académicos en los institutos de investigación así como en las universidades, que otrora eran en su mayoría ocupados por investigadores clínicos con experiencia asistencial, están siendo copados paulatinamente por investigadores básicos, en su mayoría no-médicos ( ). Al mismo tiempo, la investigación pacienteorientada está en caída; ésta actualmente "contribuye" sólo con $10 \%$ de los gastos totales del Instituto Nacional de Salud Norteamericano (NHI), cuyos gastos ascienden a casi catorce mil millones de dólares $\left.{ }^{8}\right)$. Todo ello ha llevado a afirmar que la investigación biomédica, especialmente la clínica, está en crisis $\left(^{5,8}\right)$.

Lo anterior podría indicar que el desarrollo de la investigación biomédica no se caracteriza por un desarrollo muy sistemático y organizado, sino por un desarrollo acumulativo y algo "errático". El análisis del "desarrollo epistemológico" de la medicina nos lleva a discutir y plantear políticas de desarrollo; su análisis plantea una tarea filosófica. Se puede plantear diferentes "posiciones", desde la dirigista y mecenista hasta la de laissez faire científico, que deja libertad total. Aunque, quizá adoptar una política de desarrollo científico que conserve la libertad creadora, así como el estímulo y la corrección de los desarrollos unilaterales, es decir, que sea sistemista sea lo más deseable.

La ciencia es un sistema, tanto al nivel conceptual como al nivel social. Por lo tanto, no es posible desarrollar uno cualesquiera de sus componentes si no se desarrollan los demás, más aun si consideramos que la investigación clínica es una ciencia aplicada, por ello dependiente de la investigación básica; no obstante, la "hipertrofia" exagerada de la última podría desestabilizar el sistema.

El desarrollo de una ciencia o tecnología consiste en el aumento del volumen y de la calidad de la producción científica. Al respecto, se puede decir que la medicina, desde que se refundó hace casi dos siglos, acude demasiado al dato, la "evidencia", la investigación empírica y a la "experiencia sensible", como si éstas fueran las únicas válidas y suficientes. Los datos, cálculos y operaciones empíricas, aunque indispensables, no contribuyen necesariamente a la profundidad y solidez de una ciencia o tecnología $\left({ }^{\circ}\right)$. La profundidad y madurez no son, pues, cuestión de número. Tienen que ver, además, con estructuras metacientíficas, es decir, con estructuras teóricas, con postulados y presupuestos, los cuales predeterminan y caracterizan toda ciencia; lamentablemente, en medicina la investigación teórica es bastante humilde.

Todavía tenemos mucho que hacer a fin de que nuestra medicina sea más profunda y más madura. Debemos empezar a perder el miedo a la introducción de hipótesis que contengan factores no observables, debemos empezar a luchar por la organización lógica de los postulados. Es necesario filosofar, pero no sólo en la parcela de lo ético y axiológico; la medicina plantea problemas filosóficos, desde los relacionados a las políticas científicas hasta los relacionados al análisis ontológico, lógico y metodológico. En ese sentido, 
el presente ensayo asume como pretensión principal llamar la atención a nosotros los médicos. Aspira mostrar que de atrevernos, "algunos" problemas médicos pueden ser tratados filosóficamente.

\section{La medicina basada en evidencias y su lugar en la práctica médica}

La medicina basada en evidencias (MBE) se ha convertido, según sus difusores, en el nuevo paradigma de la medicina mundial $\left({ }^{10,11}\right)$. Sus propulsores señalan que: "es una nueva propuesta de enseñanza y práctica de la medicina” $\left({ }^{12}\right)$.

"Una propuesta novedosa que plantea una saludable subversión en la medicina" $\left({ }^{13}\right)$. Casi todos ellos están de acuerdo que la MBE es una nueva forma de hacer medicina, diferente de la clínica clásica de los médicos centenarios. Empero, ¿puede ser nuevo declarar en pleno siglo XXI, tras 400 años de ciencia, que la mejor evidencia disponible y "usable" es aquella que emplea el método científico en su descubrimiento? ¿Puede ser nuevo mencionar que los ensayos clínicos -es decir, la investigación científica- tienen mayor valor de "evidencia" que la experiencia, el arte o el "instinto" clínico? ¿Puede ser nuevo señalar que se debe recurrir a la ciencia si se quiere conocer la verdad o al menos aproximarse a ella?

Lo anterior podría indicar que, a diferencia de otras ciencias fácticas, la medicina se muestra desfasada. La aparición reciente de la MBE, así como su gran publicidad e importancia aparente, significaría que recién en medicina, sobretodo en la medicina clínica asistencial, se comienza a dar importancia al "cientificismo".

La palabra paradigma, «introducida» a la literatura científica y filosófica por Thomas Kuhn, fue definida por él de múltiples formas (algún crítico le reclamó hasta veintidós formas distintas de uso). Para Kuhn, el constructo paradigma debe ser entendido al menos en dos sentidos distintos. Por una parte, significa toda la constelación de creencias, valores, técnicas, etc., que comparten los miembros de una comunidad científica. Y, por otra parte, denota una especie de elemento de tal constelación, las concretas soluciones de problemas que, empleadas como modelos o ejemplos, pueden remplazar reglas explícitas como base de solución de los restantes problemas de la ciencia (pp. 269) $\left({ }^{14}\right)$. Mario Bunge (pp. 75-78) $\left({ }^{15}\right)$, en una crítica que le hace a Thomas Kuhn, ha propuesto una definición de paradigma más clara y práctica; según Bunge, se debe entender por paradigma a un cuerpo de conocimientos ya admitidos, unidos a una hipótesis central, una problemática, un objetivo y una metódica.

Si consideramos esas definiciones, la MBE parece cumplir los requisitos de paradigma. No obstante, en términos kuhneanos para que un paradigma sea nuevo, éste tiene que ser «inconmensurable» respecto al paradigma previo. Es decir, que la transición de un paradigma en crisis a otro nuevo del que pueda surgir una nueva tradición de ciencia, está lejos de ser un proceso de acumulación, al que se llegue por medio de una articulación o una ampliación del antiguo paradigma. Es, mas bien, una reconstrucción del campo, a partir de nuevos fundamentos, reconstrucción que cambia algunas de las generalizaciones teóricas más elementales del campo (pp. 139) $\left({ }^{14}\right) \mathrm{Si}$ consideramos estas afirmaciones, podemos darnos cuenta que la MBE no puede, entonces, constituir un nuevo paradigma, ya que su fundamento central, simplemente, es una afirmación gnoseológica común a toda ciencia y tecnología, fundamento ya asumido tácitamente por la medicina pre-MBE.

Creo que no es necesario sobredimensionar ni exagerar. El principal valor de la MBE es el de ser una herramienta, básicamente, una herramienta técnica que trata de cuantificar y calificar cuál dato o cuál estudio tiene menor error $\mathrm{y}$, por tanto, mayor validez; una herramienta técnica que nos aclara la mejor forma de usar y aplicar "la evidencia". Sin embargo, no debe significar más que eso, si no queremos construir una medicina desarticulada, parcial y hasta tuerta, basada sola y casi exclusivamente en un único "paradigma".

Muchas críticas $\left({ }^{16}\right)$ contra la MBE están surgiendo, en parte por que no es juzgada en su 
verdadera dimensión, sólo como herramienta técnica; la MBE no reemplaza el proceso de razonamiento clínico, por ejemplo. Los buenos médicos, según la evidencia científica, ${ }^{17-18}$ usan su juicio personal para afirmar algo válido o no, los médicos no aplican las generalizaciones de los lineamientos clínicos cual tabla rasa, sino que lo hacen utilizando su juicio personal, determinado por el contexto clínico.

Tanenbaum $\left({ }^{17}\right)$ puntualiza que el exceso de confianza en la MBE es motivado por la creencia en la superioridad práctica del conocimiento estadístico frente a otros tipos de conocimiento. Según él, los adherentes al "nuevo paradigma" esperan suplantar el uso de la intuición, la experiencia clínica no-sistemática y el razonamiento causal (fisiológico) por el uso (aplicación) directo de la evidencia de la investigación clínica. La estadística es sólo una herramienta auxiliar, una técnica auxiliar del método científico, nos recuerda el profesor Bunge $\left({ }^{15}\right)$; la estadística no precedió a la "invención" del método científico, sino que fue posterior, su finalidad es corregir y medir el error, no dar las pautas para el conocer. De forma semejante, en medicina, la estadística también es una herramienta, no constituye "una forma de conocimiento". Empero, dar el correcto lugar a la estadística no significa hacer concesión a la intuición, a la subjetividad extrema y a la irracionalidad. Es cierto que el razonamiento clínico se mueve en medio de la incertidumbre y el caso específico $\left({ }^{18,19}\right)$, pero éste no es idiosincrásico ni difuso.

\section{La caracterización de la medicina como saber y praxis}

Parte de la organización y sistematización de la medicina es la conceptualización de la medicina misma. ¿Qué es la medicina? ¿Es arte, es ciencia o es tecnología? Deben preguntarse muchos estudiantes al enfrentarse a sus primeros años de práctica clínica. Probablemente, algunos estarán de acuerdo (al menos inicialmente) con aquello referido por sus maestros: "la medicina es arte, hijo, es el arte del diagnóstico y el arte de la terapéutica". Sin embargo, es posible, que pronto algunos perciban el sabor a ciencia, al leer los artículos médicos, al probar del rigor y generalidad de muchas de las definiciones y enunciados. Es posible, entonces, que sientan cierto desasosiego con la respuesta del maestro, y se pregunten nuevamente ¿Dónde encuentra mi maestro la característica que le permite definir a toda la medicina como arte? ¿Quizá en su forma peculiar de diagnostico? ¿Quizá en su forma única y propia de proveer tratamiento? ¿Pero si esto es así, si cada médico tiene su propio método de diagnosticar y tratar, cuál puede ser el válido, cuál puede ser el mejor, o es que todos son iguales de válidos y de buenos, y a mí, cuánto tiempo me tomará llegar a adquirir mi propio método?

Si ese estudiante poseyera mayor voluntad de encontrar respuestas, poco a poco se daría cuenta que la medicina no puede ser esencialmente una ciencia; porque aprenderá que su primordial función es tratar de salvar vidas y no la de encontrar regularidades o leyes científicas; hará uso de ellas, claro está, pero su función sine qua non, no será la de encontrarlas. Descubrirá que la medicina no puede ser un arte, ya que se rige por generalizaciones y por leyes, no por criterios singulares y particulares (como lo hace el arte); descubrirá que no podemos trabajar de forma errática, sin metodología y sin patrones repetibles. Finalmente, descubrirá que la medicina es una tecnología, es decir, una técnica usuaria del conocimiento científico.

\section{El concepto general de enfermedad}

Si bien es cierto que el médico puede caracterizar con precisión y diagnosticar con certidumbre razonable algunos o muchos problemas médicos, creo que, también es cierto que la medicina aún no posee un concepto general, claro y adecuado de enfermedad. No me refiero a un mero concepto literal o descriptivo -como el escaso concepto de enfermedad propuesto por la OMS- sino a un concepto que se engarce dentro de una teoría general de salud-enfermedad, que haga uso de condicionantes lógicos y axiomáticos. Somos testigos que tal "constructo" no existe, 
existen criterios y herramientas diagnósticas, además de principios fisiopatológicos que ayudan a identificar un proceso patológico que consideramos anormal; pero no poseemos un modelo teórico; a lo sumo existen discusiones literarias sobre la calidad valorativa, "difusa" o naturalista del concepto enfermedad $\left({ }^{20,21}\right)$.

Las discusiones de cómo definir enfermedad continúan alrededor de la vieja dicotomía naturalista-valorativa $\left({ }^{20}\right)$. Autores, como De Vitto $\left({ }^{22}\right)$, Rudnick $\left({ }^{23}\right)$ y Scadding $\left({ }^{4}\right)$, persisten en afirmar lo señalado por Canguilhem hace casi cincuenta años, esto es, que la designación de un estado saludable o patológico es dependiente de la imposición de reglas y convenciones de una sociedad $\left({ }^{2}\right)$. De forma opuesta, Boorse sigue confiando en definir enfermedad como un estado natural, libre de condicionantes normativas o de valor, confianza ya declarada un siglo atrás por Bernard $\left({ }^{20}\right)$.

Las definiciones de enfermedad elaboradas utilizando lógicas estrictas y no sólo prosa literaria son pocas; la "fuzzy disease" de Sadegh-Zadeh ${ }^{25}$ ) es hasta ahora el único ejemplo. Los teóricos de la medicina actual tampoco han acudido a la teoría general de sistemas para conseguir una definición general de enfermedad $\left({ }^{21}\right)$. Mario Bunge ya trazó el camino, ${ }^{26}$ pero parece no ser seguido. El materialismo emergentista tampoco parece haber sido oído; el único intento de su uso para definir enfermedad se plantea en uno de mis artículos $\left({ }^{27}\right)$.

\section{La epistemología personal y el proceso clínico}

Evidencia disponible muestra que la actitud de los médicos hacia los protocolos y lineamientos clínicos no es la esperada. Por ejemplo, un estudio $\left.{ }^{28}\right)$ encontró que sólo $50 \%$ de los encuestados (pediatras) conocía los lineamientos para el diagnóstico y tratamiento de otitis; adicionalmente, este estudio reportó que casi un tercio de los pediatras declaró percibir a los protocolos como "recetas de cocina". Otros estudios $\left({ }^{2,30}\right)$ señalan que la adherencia a la MBE todavía es bastante baja.

Respecto a los hallazgos anteriores, varias explicaciones han sido expuestas; éstas estriban desde la falta de tiempo y la poca difusión (aunque ya demande varios millones de dólares) $\left({ }^{28-30}\right)$ hasta aquellas que apelan a la peculiaridad del trabajo clínico. Según estas últimas, "cada médico acumularía un vasto e idiosincrásico conocimiento, conduciendo sus decisiones gracias a sus habilidades cognitivas aprendidas de la experiencia diaria, más que de la información científica literal" $\left({ }^{16}\right)$. Sin embargo, pueden existir otras razones que no han sido abordadas; una de ellas se ancla en las llamadas "creencias epistemológicas" (epistemological beliefs).

Las creencias epistemológicas conforman un conjunto de premisas y presuposiciones personales acerca del conocimiento y del aprender ${ }^{(1,32}$ ). Considerable evidencia científica ${ }^{31}$ sustenta el hecho que tal constructo, acuñado por psicólogos y educadores, juega un importante rol en el entendimiento del proceso cognitivo. Según el modelo actual, las "creencias epistemológicas" deben ser entendidas como un conjunto de creencias y actitudes que integran una teoría individual acerca de la naturaleza, certeza, origen y justificación del conocimiento. Esta teoría individual "ilustra" que las ideas de las personas acerca del conocimiento forman una estructura continua y conexa, aunque no necesariamente formal y explicita -como en el caso de un filósofo profesional ${ }^{(1)}$ ).

La evidencia, además, muestra que existen diferencias estables, claramente identificables, en las creencias de los individuos acerca del conocimiento, logrando identificarse varias "dimensiones" ${ }^{31,32}$ ). Por ejemplo, muchos individuos confían más en el conocimiento adquirido de una autoridad externa; otros en cambio, confían más en su propio juicio y otros en el producto de la interacción entre la autoridad y su contexto. Algunos individuos creen que el conocimiento es tentativo, no final; otros, en cambio que es final y poco modificable. Acerca del conocimiento de la naturaleza, algunos creen que el conocimiento es universal, aplicable a diferentes medios; otros, que es relativo, dependiente de la subjetividad e influencia externa. 
En un estudio ejecutado en Lima $\left({ }^{33}\right)$, encontramos que las creencias de los médicos acerca del conocimiento parecen mostrar una dimensión relativista (según esta "dimensión" el conocimiento es posible, pero nadie es el poseedor de la verdad, cada cual es dueño de su opinión, no existiendo verdades universales). Esta postura podría influir o reforzar una conducta profesional donde la experiencia individual es la directriz. Si las creencias epistemológicas de los médicos muestran una dimensión relativista, es posible cierto conflicto frente a la actitud realista-autoritaria asumida por quienes impulsan una medicina basada en el uso de lineamientos clínicos (de aplicación universal). Aquello puede explicar la actitud $\mathbf{m}$ muy positiva por parte de los médicos tanto hacia la MBE como al uso de lineamientos clínicos. Lamentablemente, no existen estudios dirigidos a conocer la epistemología individual del personal médico; he aquí un problema científico a investigar.

\section{Medicina alternativa ¿Realmente medicina complementaria?}

En el curso de las últimas décadas han reaparecido en el sistema de salud terapias y disciplinas que algunos creíamos estaban enterradas por la revolución científica. En aras de la apertura mental y la tolerancia, la mal llamada "medicina occidental" está tratando de ser "integrada" con disciplinas antes llamadas alternativas y hoy complementarias.

La medicina no convencional está creciendo exponencialmente; ya no es patrimonio de sociedades con historia cultural tradicional; su uso está muy extendido tanto en el mundo industrializado como en el preindustrial. En los Estados Unidos se ha determinado que el número de visitas a los "consultorios" de medicina alternativa alcanza los 425 millones, cifra que supera ampliamente el número de visitas a los consultorios médicos convencionales (388 millones) $\left({ }^{34}\right)$. Además, se ha estimado que los norteamericanos gastan aproximadamente 13700 millones de dólares al año en servicios de medicina alternativa $\left({ }^{34}\right)$, cifra que, asombrosamente, representa casi la mitad de todo el gasto en servicios médicos de los Estados Unidos.
Como vemos, el interés actual respecto a la medicina alternativa es alto $\left({ }^{35}\right)$ Esto es debido, en parte, a la supuesta "validación" reciente de las terapias alternativas por parte de la ciencia. La cantidad de investigación biomédica sobre medicina alternativa está en aumento, el número de ensayos aleatorios se duplica cada quinquenio $\left.{ }^{36}\right)$. La conocida base de datos Cochrane Library incluye hasta la actualidad cerca de cuatro mil estudios referidos a medicina alternativa $\left.{ }^{37}\right)$.

Pero, lo que alegra y alienta a muchos seguidores de las medicinas alternativas, no son sólo el número creciente de estudios publicados, sino sus resultados. Por ejemplo, un meta-análisis ${ }^{38}$ ) reciente ha mostrado mayor eficacia terapéutica "estadísticamente significativa" de algunos tratamientos homeopáticos en comparación al placebo. Esos resultados están derrumbado la vieja confianza de muchos materialistas ingenuos, quienes afirmaban que los "efectos" de las terapias alternativas eran debidos al efecto placebo.

Frente a esa avalancha de "pruebas", quizá la filosofía proporcione alguna visión más crítica y certera. Karl Popper nos enseña que, contrario a lo que pueda pensar cualquier materialista o positivista ingenuo, la confirmación repetida de efectos o hipótesis no asegura la veracidad de una teoría, ya que esta metodología no es la fundamental en la ciencia. Así, la "evidencia" por confirmación no validaría un conjunto de creencias, en este caso, un conjunto de creencias acerca de la terapéutica y diagnóstico de determinados males. En su obra "La Lógica de la Investigación Científica" $\left({ }^{9}\right)$, Popper demuestra que la confirmación por casos, como herramienta de validación, no es viable, aún apelando a las probabilidades. Dado que el universo es infinito o casi infinito (bastando el tiempo para ello), las veces que se puede repetir una observación puede ser también infinita; sin embargo, la capacidad humana para ejecutar estas observaciones es muy limitada. Si intentáramos expresar la confirmación de una observación en términos de probabilidades, la probabilidad que podríamos obtener al dividir el número de observaciones realizadas por el número 
de repeticiones posibles, sería paradójicamente muy cercana a cero.

Según Popper, es la falsabilidad la que nos asegura estar más cerca de la verdad: "Los elementos de juicio confirmatorios sólo deben ser tomados en cuenta cuando son el resultado de un genuino «test» de la teoría; es decir, cuando puede ofrecerse un intento serio, pero infructuoso, de refutar la teoría" $\left({ }^{40}\right)$.

Una teoría, para que sea científica y contrastable, debe expresar claramente enunciados prohibitivos, es decir, enunciados que nieguen la existencia de observaciones no compatibles con la teoría, de tal forma que, si se encontrasen, refuten la teoría.

El conjunto de medicinas alternativas no constituyen teorías sistemáticas que expresen enunciados prohibitivos; son por ello «teorías» no contrastables. Además, su ontología no naturalista y su gnoseología no realista impiden la contrastación de sus enunciados. Por último, sus efectos empíricos no han sido contrastados, a pesar de la cantidad de estudios que confirmarían sus efectos; por ejemplo, en el meta-análisis mencionado sobre tratamientos homeopáticos $\left({ }^{38}\right)$ se muestra que en ningún caso un estudio ha sido replicado, es decir, fue ejecutado por al menos tres investigadores independientes; de forma semejante, en el caso de otras medicinas alternativas, los estudios son contradictorios, algunos a favor y otros en contra. La calidad de los estudios muestra deficiencias; en el caso de la medicina herbal la cantidad y calidad de estudios aleatorios placebo controlados es mínima y no concluyente ¿Por qué se debe aceptar, entonces, la validez de estas terapias?

\section{Conclusión}

Los apartados que precedieron, no son más que algunos ejemplos de la cantidad de problemas que se puede abordar agregando alguna dosis de filosofía a nuestra tarea científica. La medicina ofrece, pues, formidables problemas a escarbar. Esto muestra que la filosofía no le es extraña ni a la práctica médica ni a la medicina como teoría; así pues, a poco que se examine, la filosofía ofrece a la medicina una visión crítica pero a la vez estimulante y constructiva, sin resumirse sólo a la parcela de lo ético, lo axiológico o lo histórico.

\section{BIBLIOGRAFÍA}

1. Regan-Smith M. Reform without change: update, 1998. Acad Med 1998; 73: 505-7.

2. Aréchiga H. Educación médica continua. Gac Méd Mex 1996; 132 (Supl. 2): 141-3.

3. National Library of Medicine. Disponible en: http:// www.ncbi.nlm.nih.gov. (Ingresado en abril del 2002).

4. Weiner JM, Shirley S, Gilman NJ, Stowe SM, Wolf RM. Access to data and the information explosion: oral contraceptives and risk of cancer. Contraception 1981;24: 301-13.

5. Schechter A. The crisis in clinical research: Endangering the half-century National Institutes of Health Consensus. JAMA 1998; 280: 1440-2.

6. Gill GN. The end of the physician scientist. Am Scholar 1984; 53: 353-68.

7. Wilmshurst P. Devaluing clinical skills. BMJ 2000; 320 : 1739.

8. Ahrens EH. The Crisis in Clinical Research: Overcoming Institutional Obstacles. New York, NY: Oxford University Press; 1992.

9. Bunge MA, García JL, SempereJ. (trad.) Teoría y Realidad. Barcelona: Ariel 1981; 89-107.

10. Sackett D, Rosenberg WM, Gray JAM, Haynes RB, Richardson WS. Evidence based medicine: what it is and what it isn't. BMJ 1996; 312: 71-2.

11. Knottnerus JA, Dinant GJ. Medicine based evidence, to prerequisite for evidence based medicine. BMJ 1997; 315: 1109-10.

12. Evidence-Based Medicine Working Group. Evidencebased medicine: a new approach to teaching the practice of medicine. JAMA 1992; 268: 2420-5.

13. Huicho L. La medicina basada en evidencias como un nuevo paradigma de la enseñanza y la práctica de la medicina. An Fac Med 1997; 58(2): 118-27.

14. Kuhn T, Contin A (trad.). La Estructura de las Revoluciones Científicas. Bogotá: Fondo de Cultura Económica; 1998. Originalmente publicado en inglés: The Structure of Scientific Revolutions. Chicago: The University of Chicago Press; 1962.

15. Bunge MA. Vigencia de la Filosofía: Ciencia y técnica. Investigación y universidad. Lima: Universidad Inca Garcilaso de la Vega, Fondo editorial; 1997.

16. Rosoff A. Evidence-based medicine and the law: the courts confront clinical practice guidelines. J Health Politics, Policy and Law 2001; 26: 327-68.

17. Tanenbaum SJ. What physicians know. NEngl J Med 1993; 
329: 1268-71.

18. Naylor CD. Grey zones of clinical practice: some limits to evidence-based medicine. Lancet 1995; 345: 840-2.

19. Beresford EB. Uncertainty and the shaping of medical decisions. Hastings Cent Rep 1991; 21: 6-11.

20. Peña A, Paco O.El concepto general de enfermedad. Revisión, crítica y propuesta. Primera parte. Anales de la Facultad de Medicina 2002; 63: 223-32.

21. Peña A, Paco O.El concepto general de enfermedad. Revisión, crítica y propuesta. Segunda parte: carencias y defectos en los intentos por lograr una definición general de enfermedad. An Fac Med 2002; 63: 313-21.

22. De VittoS. On the value-neutrality of the concepts of health and disease: unto the breach again. J Med Philos 2000; 25: 539-67.

23. Rudnick A. The ends of medical intervention and the demarcation of the normal from the pathological. J Med Philos 2000; 25: 569-80.

24. Scadding JG. Essentialism and nominalism in medicine: logic of diagnosis in disease terminology. Lancet 1996; 348: 594600.

25. Sadegh-Zadeh K. Fuzzy health, illness, and disease. J Med Philos 2000; 25: 605-38.

26. Bunge MA. Epistemología. Curso de actualización. Barcelona: Ariel; 1980: 238-243.

27. Peña A, Paco O. El concepto general de enfermedad. Tercera parte: Un modelo teórico de enfermedad. An Fac Med 2003; 64(1): 55-62.

28. Christakis D, Rivara F. Pediatricians' awareness of and attitudes about four clinical practice guidelines. Pediatrics 1998; 101: 825-30.

29. McColl A, Smith H, White P. General practitioners' perceptions of the route to evidence based medicine: a questionnaire survey. BMJ 1998; 316: 361-5.

30. Olatunbosun O, Edouard L, Pierson R Physicians' attitudes toward evidence based obstetric practice: a questionnaire survey. BMJ 1998; 316: 365-6.

31. Hofer BK, Pintrich PR. The development of epistemological theories: Beliefs about knowledge and knowing and their relation to learning. Rev Educat Res 1997; 67: 88-140.

32. Hammer D, Elby A. Epistemological Resources. En: Fishman B, O'Connor-Divelbiss S (Eds.) Mahwah, NJ: Erlbaum. Fourth International Conference of the Learning Sciences 2000: 4-5.

33. Peña A, Paco O, Peralta C. Epistemological beliefs and knowledge among physicians: A questionnaire survey. Medical Education Online 2002; 7: 4. Disponible desde URL http://www.med-ed-online.org.

34. Eisenberg DM, Kessler RC, Foster C, Norlock FE, Calkins DR, Delbanco TL. Unconventional medicine in the United States - prevalence, costs, and patterns of use. N Engl J Med 1993; 328: 246-52.

35. Goldbeck-Wood S. Medicine and the media: Complementary medicine and the cure for credulity. BMJ 1996; 312: 1678.
36. Vickers AJ. Bibliometric analysis of randomised controlled trials in complementary medicine. Complementary Ther Med 1998; 6: 185-9.

37. Cochrane Collaboration and the Cochrane Library. Disponible en http://www.cochrane.org/(Ingresado en Enero del 2003).

38. Linde K. Clausius N, Ramirez G, Melchart D, Eitel F, Hedges LV, et al. Are the clinical effects of homeopathy placebo effects? A meta-analysis of placebo controlled trials. Lancet 1997; 350: 834-43.

39. Popper KR, Sánchez de Zavala (trad.). La Lógica de la Investigación Científica. Madrid; Tecnos; 1962. Originalmente publicado, en inglés: The Logic of Scientific Discovery, London; Hutchinson; 1959.

40. Popper KR. El Desarrollo del Conocimiento Científico, Conjeturas y Refutaciones. Barcelona; Paidos 1965: 47. 